\title{
Erratum to: Statistical Entropy of Vaidya-de Sitter Black Hole to All Orders in Planck Length
}

\author{
HangBin Sun · Feng He · Hai Huang
}

Published online: 23 September 2012

(C) Springer Science+Business Media, LLC 2012

Erratum to: Int. J. Theor. Phys. (2012) 51:1762-1768

DOI 10.1007/s10773-011-1053-y

We regret that an initially typographical error in the equations, Eq. (15) of HangBin Sun (2012), has propagated through the paper. Corrections are as follows:

$$
\begin{aligned}
p^{2} & =-\omega p_{r}+f p_{r}^{2}+\frac{1}{r^{2}} p_{\theta}^{2}+\frac{1}{r^{2} \sin ^{2} \theta} p_{\varphi}^{2}=\omega p_{r}, \\
p^{2} & =\omega \frac{p_{r+}+p_{r-}}{2}=\frac{\omega^{2}}{f}, \\
g(\omega) & =\frac{1}{(2 \pi)^{3}} \int e^{-\lambda p^{2}} d r d \theta d \varphi d p_{r} d p_{\theta} d p_{\varphi} \\
& =\frac{2}{(2 \pi)^{3}} \int e^{-\lambda p^{2}} d r d \theta d \varphi \int \frac{1}{\sqrt{f}}\left(\frac{\omega^{2}}{f}-\frac{1}{r^{2}} p_{\theta}^{2}-\frac{1}{r^{2} \sin ^{2} \theta} p_{\varphi}^{2}\right)^{\frac{1}{2}} d p_{\theta} d p_{\varphi} \\
& =\frac{2 \omega^{3}}{3 \pi} \int e^{-\frac{\lambda \omega^{2}}{f}} \frac{r^{2}}{f^{2}} d r,
\end{aligned}
$$

The online version of the original article can be found under doi:10.1007/s10773-011-1053-y.

H. Sun $\cdot$ F. He $(\bowtie) \cdot$ H. Huang

College of Physics and Electronic Science, Hunan University of Science and Technology,

XiangTan 411201, Hunan Province, People's Republic of China

e-mail: fhe@hnust.edu.cn

H. Sun

e-mail: sunhblw@126.com 


$$
\begin{aligned}
& F(\beta)=\frac{1}{\beta} \int d g(\omega) \ln \left(1-e^{-\beta \omega}\right) \\
& =-\frac{2}{3 \pi} \int e^{-\frac{\lambda \omega^{2}}{f}} \frac{r^{2}}{f^{2}} d r \int_{0}^{\infty} \frac{\omega^{3} d \omega}{e^{\beta \omega}-1}, \\
& S_{+}=\beta_{+}^{2} \frac{\partial F}{\partial \beta_{+}} \\
& =\frac{2 \beta_{+}^{2}}{3 \pi} \int_{r_{+}}^{r_{+}+\varepsilon_{+}} e^{-\frac{\lambda \omega^{2}}{f}} \frac{r^{2}}{f^{2}} d r \int_{0}^{\infty} \frac{e^{\beta_{+} \omega} \omega^{4} d \omega}{\left(e^{\beta_{+} \omega}-1\right)^{2}} \\
& =\frac{2 \beta_{+}^{-3}}{3 \pi} \int_{r_{+}}^{r_{+}+\varepsilon_{+}} e^{-\frac{\lambda \omega^{2}}{f}} \frac{r^{2}}{f^{2}} d r \int_{0}^{\infty} \frac{e^{x} x^{4} d x}{\left(e^{x}-1\right)^{2}}, \\
& \int_{r_{+}}^{r_{+}+\varepsilon_{+}} e^{-\frac{\lambda \omega^{2}}{f}} \frac{r^{2}}{f^{2}} d r=\int_{r_{+}}^{r_{+}+\varepsilon_{+}}\left(-\frac{r^{2}}{f^{\prime}}\right)\left(-\frac{1}{f^{2}}\right) f^{\prime} e^{-\frac{\lambda \omega^{2}}{f}} d r, \\
& \int_{r_{+}}^{r_{+}+\varepsilon_{+}} e^{-\frac{\lambda \omega^{2}}{f}} \frac{r^{2}}{f^{2}} d r \approx-\frac{r_{+}^{2}}{2 \kappa\left(1-2 \dot{r}_{+}\right)} \int_{r_{+}}^{r_{+}+\varepsilon_{+}}\left(-\frac{1}{f^{2}}\right) f^{\prime} e^{-\frac{\lambda \omega^{2}}{f}} d r \\
& =-\frac{r_{+}^{2}}{2 \kappa\left(1-2 \dot{r}_{+}\right)} \int_{f^{-1}\left(r_{+}\right)}^{f^{-1\left(r_{+}+\varepsilon_{+}\right)}} e^{-\lambda \omega^{2} \frac{1}{f}} d\left(\frac{1}{f}\right) \\
& =\frac{r_{+}^{2}}{2 \kappa\left(1-2 \dot{r}_{+}\right) \lambda \omega^{2}} e^{-\frac{\lambda \omega^{2}}{2 \kappa\left(1-2 \dot{r}_{+}\right) \varepsilon+}}, \\
& S_{+}=\frac{2 \beta_{+}^{-3}}{3 \pi} \int_{0}^{\infty} \frac{r_{+}^{2}}{2 \kappa\left(1-2 \dot{r}_{+}\right) \lambda \omega^{2}} e^{-\frac{\lambda x^{2}}{2 \kappa\left(1-2 \dot{r}_{+}\right) \beta_{+}^{2} \varepsilon_{+}}} \frac{e^{x} x^{4} d x}{\left(e^{x}-1\right)^{2}} \\
& =\frac{A_{+}}{24 \pi^{3}\left(1-2 \dot{r}_{+}\right) \lambda} \int_{0}^{\infty} e^{-\frac{\lambda x^{2}}{2 \kappa\left(1-2 \dot{r}_{+}\right) \beta_{+}^{2} \varepsilon_{+}}} \frac{e^{x} x^{2} d x}{\left(e^{x}-1\right)^{2}} \\
& =\frac{C}{6 \pi^{3} \lambda} \frac{A_{+}}{4} \frac{1}{1-2 \dot{r}_{+}}, \\
& C=\int_{0}^{\infty} e^{-\frac{\lambda x^{2}}{2 \kappa\left(1-2 r_{+}\right) \beta_{+}^{2} \varepsilon_{+}}} \frac{e^{x} x^{2} d x}{\left(e^{x}-1\right)^{2}} \approx \int_{0}^{\infty} e^{-\frac{x^{2}}{2 \pi^{2} e}} \frac{e^{x} x^{2} d x}{\left(e^{x}-1\right)^{2}} \approx 2.90
\end{aligned}
$$

" $\lambda=\frac{C}{12 \pi^{3}}$ " below Eq. (28) should be changed into " $\lambda=\frac{C}{6 \pi^{3}}$ ". Numbers following each equation are the equation numbers of HangBin Sun (2012).

The changes are minute and in no way alter the conclusions of the paper. 